Artículo de investigación.

Cómo citar: I. López, H. Peña y A. Aparicio. "Modelado de procesos de negocio para la obtención y renovación de registro calificado utilizando Togaf". Inventum, vol. 15, no. 28 , pp. 33-50. doi: 10.26620/uniminuto. inventum.15.28.2020.33-50

Editorial: Corporación Universitaria Minuto de Dios - UNIMINUTO.

ISSN: $1909-2520$

elSSN: $2590-8219$

Fecha de recibido: 10 de enero de 2020 Fecha de aprobado: 20 de marzo de 2020 Fecha de publicación: 30 de mayo de 2020

Conflicto de intereses: los autores han declarado que no existen intereses en competencia.

\section{Modelado de procesos de negocio para la obtención y renovación de registro calificado utilizando Togaf}

\author{
Business process modeling for obtaining \\ and renewing qualified registration \\ using Togaf
}

\author{
Modelação de processos empresariais \\ para obtenção e renovação de registo \\ qualificado usando o Togaf
}

Iván Arturo López Ortiz ivan.lopez@unad.edu.co https://orcid.org/0000-0003-3619-0689 Universidad Nacional Abierta y a Distancia (UNAD). Colombia

Hernando José Peña Hidalgo hernand.pena@unad.edu.co http://orcid.org/0000-0002-3477-2645 Universidad Nacional Abierta y a Distancia (UNAD). Colombia

Alexandra Aparicio alexandra.aparicio@unad.edu.co http: / / orcid.org/0000-0002-7567-6510 UUniversidad Nacional Abierta y a Distancia (UNAD). Colombia

\begin{abstract}
Resumen
En la actualidad la gestión de la información es una de las actividades de gran importancia en las organizaciones y ante la necesidad de mejorarla han surgido propuestas que permiten optimizar su gestión mediante la implementación de herramientas tecnológicas que integren los procesos y la gestión humana, lo que permite encaminar el uso y aprovechamiento de la información; es así como se identificó que la Escuela de Ciencias Básicas Tecnología e Ingeniería (ECBTI) adscrita a la Universidad Nacional Abierta y a Distancia (UNAD), en la actualidad soporta gran parte de sus actividades en los procesos y procedimientos generados por la alta dirección de la universidad, establecidos con formatos procedimentales, muchos de ellos sin hacer uso de sistemas de información y con soporte tecnológico básico, basado en herramientas de gestión, lo que conlleva a demoras, y pérdida de información en determinado momento. Por lo anterior, se propone realizar un estudio de orden empresarial que ayude a organizar los objetivos de la organización y alinearlos con tecnologías de la información (TI), en este sentido el presente artículo indica cómo utilizar el marco de trabajo (framework) de The Open Group Architecture Framework (Togaf) en donde se aborda las fases iniciales como son: preliminar, visión de arquitectura, arquitectura de negocios, arquitecturas de sistemas de información y arquitectura de tecnología. Aplicados en el proceso de gestión de la oferta educativa en dos de sus procedimientos como son nuevo registro calificado y renovación de registro, en el que se identifican las brechas y propuestas de mejora, y se contribuye de esta manera con aspectos iniciales para una futura implementación de la arquitectura empresarial al interior de la ECBTI.
\end{abstract}

Palabras clave: arquitectura empresarial, gestión curricular, marco de trabajo, gestión de la calidad, TOGAF. 


\begin{abstract}
Nowadays, information management is one of the most important activities in organizations and in view of the need to improve it, proposals have arisen that allow optimizing its management through the implementation of technological tools integrating processes and human management, which allows to direct the use and exploitation of information; This is how it was identified that the School of Basic Sciences, Technology and Engineering (ECBTI), attached to the National Open and Distance University (UNAD), is currently supporting a large part of its activities from the processes and procedures generated by the university's top management, established with procedural formats, many of them without making use of information systems and with basic technological support, based on management tools, leading to delays, and loss of information at certain times. Thus, it is proposed to carry out a study of business order that helps to organize the objectives of the organization and align them with information technology (IT). In this sense, this article indicates how to use the framework of The Open Group Architecture Framework (Togaf) where the initial phases are addressed, such as: preliminary, vision architecture, business architecture, information systems architecture and technology architecture. Applied in the process of Educational Offer Management in two of its procedures such as new qualified registration and renewal of registration identifying gaps and proposals for improvement, thus contributing with initial aspects for a future implementation of the enterprise architecture within the ECBTI.
\end{abstract}

Keywords: enterprise architecture, curriculum management, framework, quality management, Togaf.

\section{Resumo}

Actualmente, a gestão da informação é uma das actividades mais importantes nas organizações, e tendo em conta a necessidade de a melhorar, surgiram propostas que permitem a optimização da sua gestão através da implementação de ferramentas tecnológicas que integram processos e gestão humana, o que permite a utilização e exploração da informação; Assim foi identificado que a Escola de Ciências Básicas, Tecnologia e Engenharia (ECBTI) ligada à Universidade Nacional Aberta e à Distância (UNAD), apoia actualmente grande parte das suas actividades nos processos e procedimentos gerados pelos quadros superiores da universidade, estabelecidos com formatos processuais, muitos deles sem recorrer a sistemas de informação e com apoio tecnológico básico, baseado em ferramentas de gestão, o que conduz a atrasos, e à perda de informação em determinados momentos. Neste sentido, este artigo indica como utilizar a estrutura do The Open Group Architecture Framework (Togaf) onde são abordadas as fases iniciais, tais como: preliminar, visão arquitectónica, arquitectura empresarial, arquitectura de sistemas de informação e arquitectura tecnológica. Aplicado no processo de gestão da oferta educacional em dois dos seus procedimentos, tais como novo registo qualificado e renovação do registo, que identifica lacunas e propostas de melhoria, contribuindo assim com aspectos iniciais para uma implementação futura da arquitectura empresarial no âmbito do ECBTI.

Palavras-chave: arquitectura empresarial, gestão curricular, enquadramento, gestão da qualidade, TOGAF. 


\section{INTRODUCCIÓN}

En la actualidad, las instituciones se enfrentan a desafíos permanentes, así como a la necesidad de mantener niveles de competitividad acordes a los retos de la globalización. Desde este punto de partida es imprescindible establecer estrategias que permitan obtener ventajas competitivas mediante la toma de decisiones acertadas, de ahí que las instituciones necesiten incorporar nuevas tendencias en su interior. Uno de ellos es la adopción de un modelo de arquitectura empresarial, el cual puede fortalecer la estructura funcional, jerárquica y de procesos.

Para el caso de la ECBTI de la UNAD, uno de los mayores retos es buscar una manera de optimizar sus procedimientos relacionados con la gestión curricular.

En este sentido se busca establecer o iniciar una arquitectura empresarial al interior de la ECBTI que soporte los objetivos de la organización y más que eso los procesos establecidos en la misma, describir la tecnología fundamental y la estructura necesaria para adecuar una estrategia tecnológica que permita fortalecer el área de tecnología de información y que sea un activo de la escuela capaz de responder a una iniciativa de negocio competitiva y exitosa.

Con lo anterior, el poder soportar mediante una Arquitectura Empresarial ( $\mathrm{AE}$ ) que permita mejorar la ruta tecnológica que en la actualidad se aplica a dos procedimientos clave en la ECBTI, como lo son la gestión de nuevos registros calificados y gestión para la renovación de registro calificado de los programas académicos en la escuela, permitirá tener un adecuado control, seguridad y disponibilidad permanente de la información.

Para lograr tal propósito y luego de revisar algunos marcos de referencia (Zachman, Togaf, DoDaf), los que proporcionan los caminos a seguir que permiten documentar la arquitectura, se empleó Togaf, que se considera como "una herramienta para asistir en la aceptación, creación, uso, y mantenimiento de arquitecturas. Está basado en un modelo iterativo de procesos apoyado por las mejores prácticas y un conjunto reutilizable de activos arquitectónicos existentes" [1], lo que permite involucrar varias áreas de la ECBTI. El modelo inicia con la identificación del estado en el que se encuentra en términos de arquitectura de negocio, arquitectura de información, arquitectura de aplicaciones y arquitectura de tecnología. Al final se muestra el avance del desarrollo tecnológico de la propuesta que permita dar una posible solución a lo planteado en el proyecto, se tiene como premisa que "la definición de principios que reflejan el consenso en toda la empresa y representan el espíritu de la arquitectura empresarial como base de la misma, requiere tener en cuenta factores de impacto para el éxito de $\mathrm{AE}$ con el fin de garantizar que han de estar presentes y con la importancia necesaria durante el ciclo de vida de la arquitectura" [2].

\section{METODOLOGÍA}

En general, la metodología consistió en seguir el ciclo de vida del método de desarrollo de la arquitectura (ADM) Togaf [3], la representación del ciclo de vida se encuentra en la figura 1 , y es una de las metodologías más populares para desarrollar $\mathrm{AE}$, que permite planificar, diseñar, evaluar e implementar una arquitectura empresarial de información en una organización, de las cuales se abordan las fases: preliminar, visión de arquitectura, arquitectura de negocio, arquitectura de sistemas de información y arquitectura de tecnología. Las siguientes fases serán caso de estudio una vez se ha definido la implementación tecnológica requerida. 


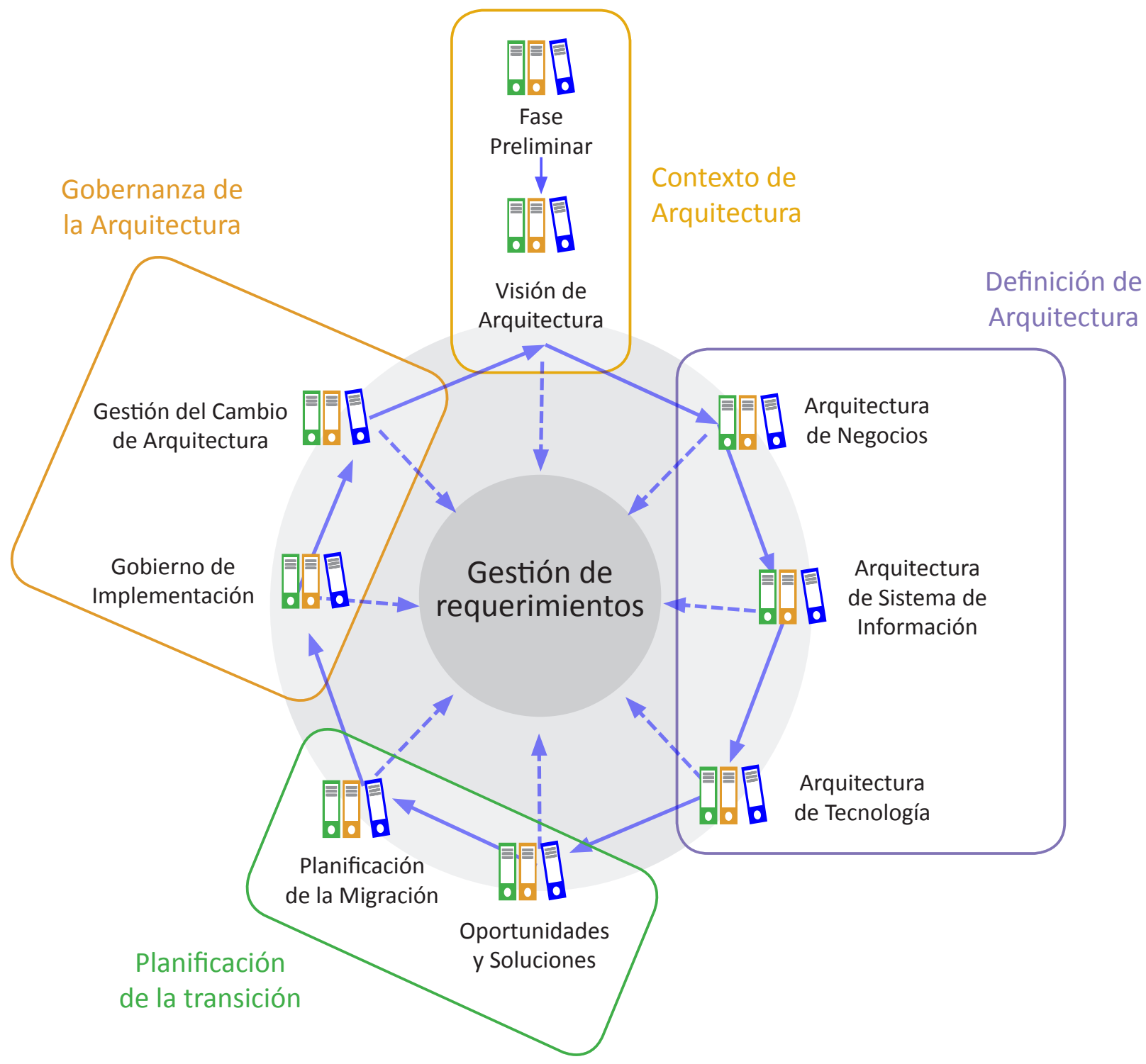

Fig. 1 Ciclo de ADM Togaf.

Fuente: elaboración propia.

\section{RESULTADOS Y DISCUSIÓN}

\section{A. Fase preliminar}

"Prepara la organización para llevar a cabo proyectos exitosos de arquitectura gracias al uso de Togaf" [4].
La figura 2 permite observar los elementos afectados por el trabajo de AE en la ECBTI.

Unidades impactadas: se identificaron las unidades de decanatura y líderes de programas académicos porque son quienes interactúan directamente con la gestión oportuna y sistémica de la información de soporte. 


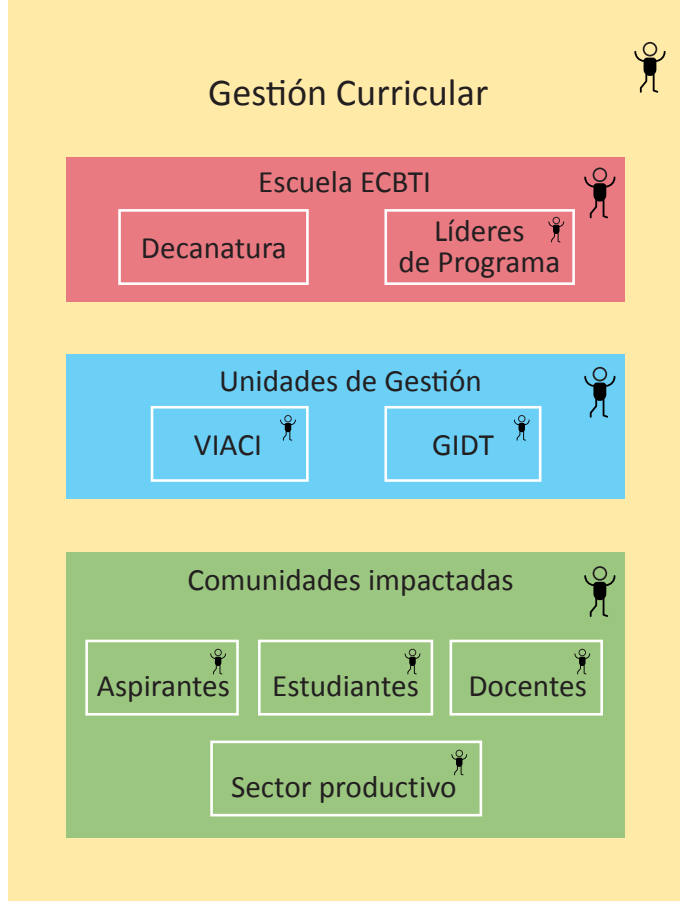

Fig. 2. Organizaciones empresariales afectadas. Fuente: elaboración propia.

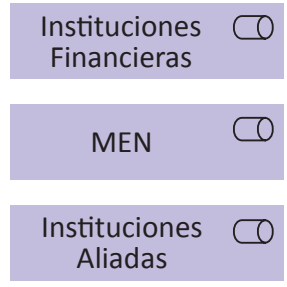

Actor Empresarial

Papel de la empresa

Ubicación

Actor Empresarial
Unidades impactadas

Unidades impactadas blandas

Unidades impactadas extendidas

Comunidades afectadas
Unidades impactadas blandas: el cambio puede afectar sus capacidades y trabajar con las unidades centrales, no se afecta directamente, para el caso la Vicerrectoría Académica y la gerencia de innovación y desarrollo tecnológico.

Unidades impactadas extendidas: están por fuera de la UNAD y no se verá afectada en su propia arquitectura empresarial.

Comunidades impactadas: se verán afectadas y se encuentran en grupos de comunidades que de una u otra manera hacen parte de los resultados de la arquitectura diseñada.

En esta fase también se destaca la importancia de tener un repositorio de arquitectura que almacene los elementos resultantes de ADM y el cual permite dar orden a los diferentes documentos que soportan la arquitectura. En este sentido se recurre a la experiencia "Aplicación de un esquema de arquitectura empresarial (Togaf) para una Pequeña Empresa (PYME) con la utilización de Aplicaciones Colaborativas de Google" [5]. Como insumo inicial para la construcción del repositorio documental de la arquitectura, en donde se aprovecharon las herramientas colaborativas de Google para apoyar los procesos internos de la organización en un periodo no mayor a un año.

Nivel de madurez: Con la identificación de la ECBTI y los principios del negocio se logró determinar los planes y estrategias del modelo motivacional, dentro de los cuales se pudo evidenciar una alta utilización de herramientas TI para diferentes procesos, cada una de ellas dispersa, para el caso de los procesos objeto de estudio, se evidenció solo la utilización de herramientas de productividad donde cada actor hace uso y dispone de ellas como él crea conveniente. Se estableció que la capacidad de negocio se debe enfocar en alinear los procesos de gestión curricular establecidos por la vicerrectoría académica y documentados en el Sistema Integrado de Gestión (SIG). Igualmente se identificó que existe una brecha en el nivel de madurez de las capacidades arquitectónicas, todas estas brechas se lograron identificar a partir de entrevistas con los líderes de programas académicos de la ECBTI como se observa en la figura 3 


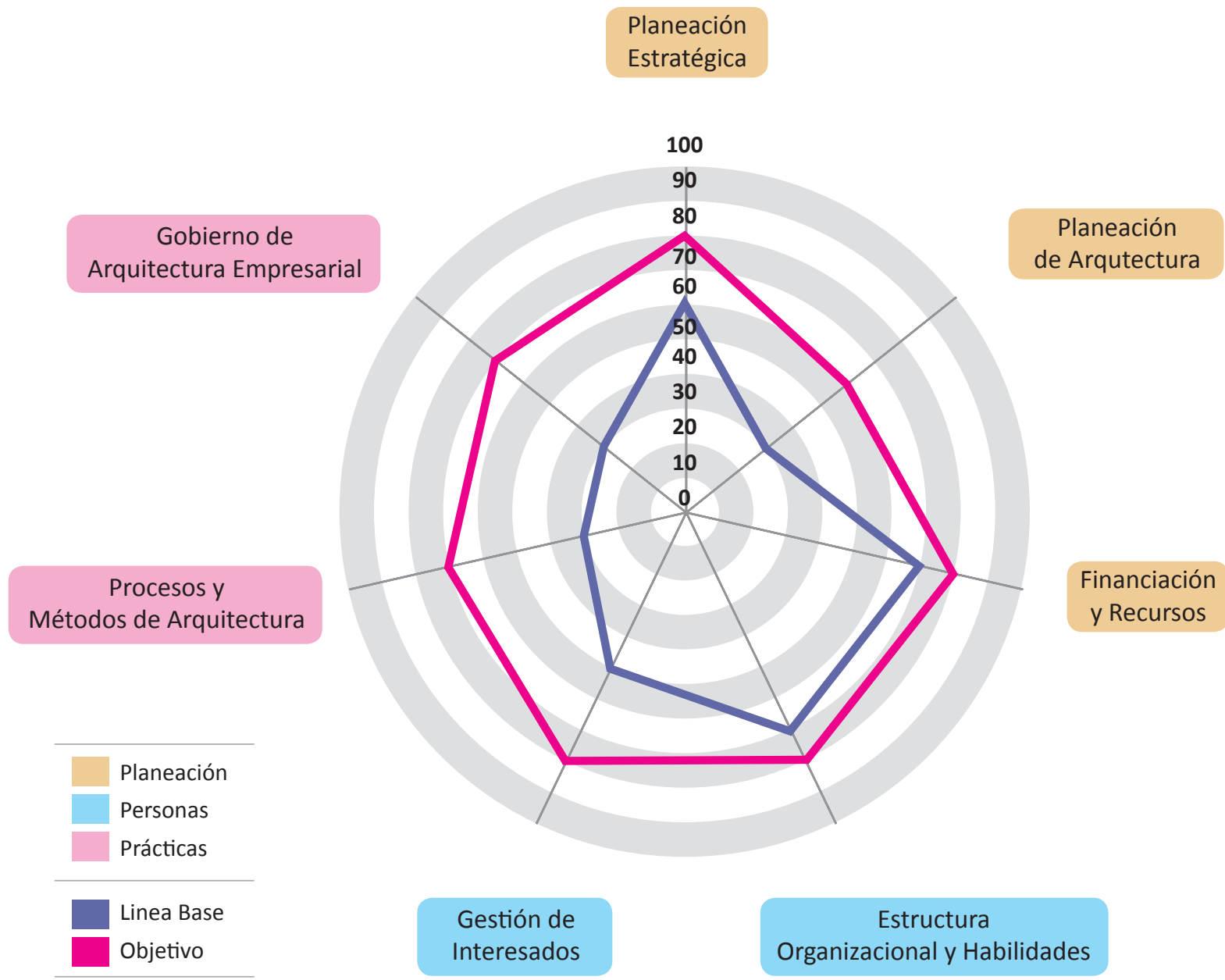

Fig. 3. Capacidades arquitectónicas afectadas.

Fuente: elaboración propia.

En este caso se busca reducir la brecha base en 20 puntos en la capacidad de planeación estratégica para lograr optimizar los procedimientos de la ECBTI para la gestión curricular; 30 puntos en planeación de arquitectura lo que permite alinear la estrategia, estructura organización, procesos, procedimientos, talento humano, recursos y valores; 10 puntos en recursos financieros; 40 puntos en procesos y métodos de arquitectura, debido a que no existe marco de iteraciones; 40 puntos en gobierno de arquitectura empresarial, dado que se requiere la construcción del marco de gobernanza; 10 puntos en estructura, organización y habilidades; 30 puntos en gestión de interesados, ya que es necesario definir de forma adecuada, los interesados y el plan de comunicaciones.

Como resultado de esta fase también se establece el cronograma de trabajo y los impulsores del negocio. Se ajustaron algunos principios y se estableció el repositorio de la arquitectura, el cual contendrá seis clases de información para la arquitectura como se describen en la tabla 1. 


\begin{tabular}{|l|l|}
\hline \multicolumn{1}{|c|}{ Clase } & \multicolumn{1}{c|}{ Descripción } \\
\hline $\begin{array}{l}\text { Arquitectura } \\
\text { metamodelo }\end{array}$ & $\begin{array}{l}\text { Describe la aplicación personalizada de un framework de arquitectura, } \\
\text { que incluye un método para el desarrollo de arquitectura y un } \\
\text { metamodelo para el contenido de arquitectura. }\end{array}$ \\
\hline $\begin{array}{l}\text { Capacidad de } \\
\text { arquitectura }\end{array}$ & $\begin{array}{l}\text { Define los parámetros, estructuras y procesos que apoyan el gobierno } \\
\text { del repositorio de arquitectura. }\end{array}$ \\
\hline $\begin{array}{l}\text { Paisaje } \\
\text { arquitectónico }\end{array}$ & $\begin{array}{l}\text { Presenta una representación arquitectónica de los activos en uso, o } \\
\text { planeados, por la empresa en puntos concretos en el tiempo. }\end{array}$ \\
\hline $\begin{array}{l}\text { Base de } \\
\text { información de } \\
\text { normas }\end{array}$ & $\begin{array}{l}\text { Captura los estándares que deben cumplir las nuevas arquitecturas, } \\
\text { puede incluir estándares de la industria, productos seleccionados } \\
\text { y servicios de proveedores, o servicios compartidos ya desplegados } \\
\text { dentro de la organización. }\end{array}$ \\
\hline $\begin{array}{l}\text { Biblioteca de } \\
\text { referencia }\end{array}$ & $\begin{array}{l}\text { Provee directrices, plantillas, patrones y otras formas de material } \\
\text { de referencia que pueden ser apalancados con el fin de acelerar la } \\
\text { creación de nuevas arquitecturas para la empresa. }\end{array}$ \\
\hline $\begin{array}{l}\text { Registro de } \\
\text { gobernanza }\end{array}$ & \begin{tabular}{l} 
Provee un registro de la actividad de gobernanza en toda la empresa. \\
\hline
\end{tabular}
\end{tabular}

Tabla I. Clases de información para la arquitectura

Fuente: elaboración propia.

\section{B.Visión de la Arquitectura}

"Se deben identificar las partes interesadas, sus inquietudes y requerimientos de negocio" [6].

Antecedentes del problema: la ECBTI, genera gran parte de su información a partir de documentos que cada uno de sus miembros tienen almacenados en sus propios repositorio como son: archivos de gestión almacenados en los discos duros de sus computadores, enlazamiento sin una identificación precisa, utilización de herramientas de almacenamiento en la nube creadas con cuentas personales como Google drive o Dropbox, sin que la Universidad tenga un control o acceso a esta información, lo que convierte al proceso de gestión de la información en una de las actividades más complejas, dado que se requiere del conocimiento y de la memoria que cada uno de los funcionarios tenga en relación con la información solicitada en determinado momento.

El "Diseño de un modelo de arquitectura empresarial para publicaciones científicas basado en ADM Togaf" [7], se toma como punto de partida para abordar esta fase porque muestra que no solo se pueden encontrar estudios directamente ligados a empresas si no en áreas específicas como la Ciberseguridad.

El proceso de gestión de la oferta educativa en sus procedimientos: gestión de nuevos registros calificados y renovación de registro calificado, donde se involucra la gestión de la información y del conocimiento organizacional es complejo, dado que también interviene la normatividad planteada por el SIG [8], los lineamientos establecidos para la sala Conaces y el CNA, además de cuestiones propias de la estructura de la ECBTI.

En este sentido, para los líderes de programas académicos es fundamental recoger todas las evidencias que se tengan en cada uno de los centros del país y si bien existe documentación en el SIG, en la Escuela no existe un documento que rija los pasos de principio a fin, en torno a recolección, análisis y tratamiento de esta información, convirtiéndose esta tarea en una necesidad de recopilación de información inmediata por parte de los líderes de programas y secretarios académicos, decanos y en 
muchas ocasiones se recurre a la memoria como fuente de información. Es de mencionar la importancia en que no es solo recolectar la información sino asegurarse de la calidad de estos documentos.

Por otra parte, el no contar con estos procesos de organización de la información puede conllevar a la pérdida histórica del ciclo de vida de un programa académico. En síntesis y siendo la gestión de la información un proceso fundamental en toda organización; es importante organizar la información al interior de la ECBTI, y hacer énfasis en las actividades de renovación y actualización curricular.

A partir de esta descripción se da paso a realizar un cambio en los conductores y las oportunidades, también a plantear los objetivos del negocio, los requisitos y la representación de alto nivel de la arquitectura de destino y cómo esto proporciona una solución a las necesidades de la empresa, para explicar el valor añadido del esfuerzo de arquitectura a los interesados como se indica en la figura 4.

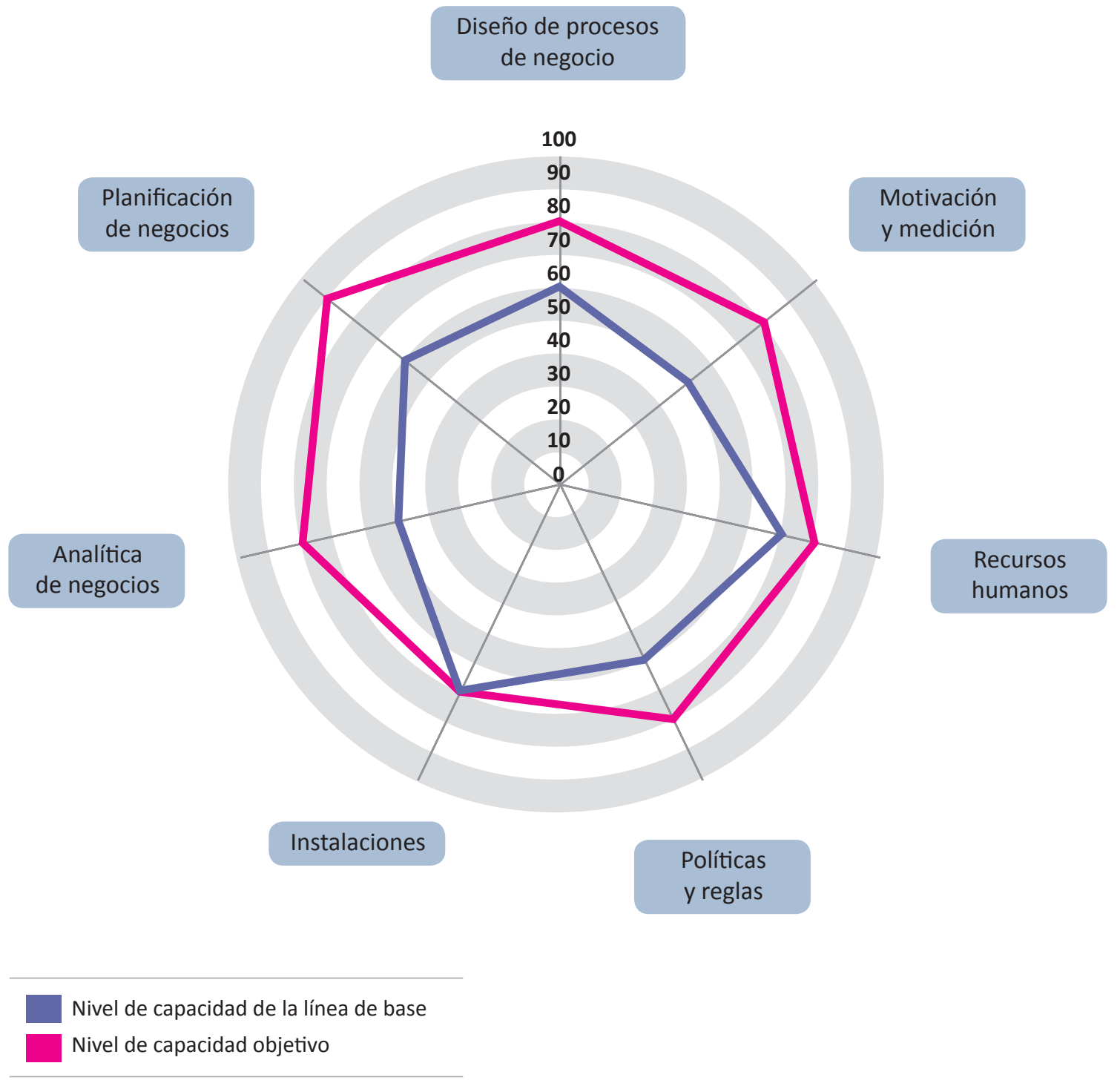

Fig. 4. Capacidades de TI para apoyar el desarrollo arquitectural. Fuente: Fuente propia. 
En este plan de evaluación se identifican las capacidades, nivel de capacidad inicial, de destino, la brecha y el enfoque que se da para reducir esta brecha. En este caso para reducir 20 puntos la brecha de la capacidad tecnológica. El diseño de procesos de negocio se debe alinear con los procesos propios de la ECBTI; 30 puntos en motivación y medición; 10 puntos en recursos humanos; 20 puntos en políticas y reglas; 0 puntos en instalaciones, lo que indica que esto está superado; 30 puntos en analítica de negocio; 30 puntos en planificación de negocios.

También se realizó la evaluación de capacidades relacionadas con $\mathrm{TI}$, la cual se puede identificar en la figura 5.

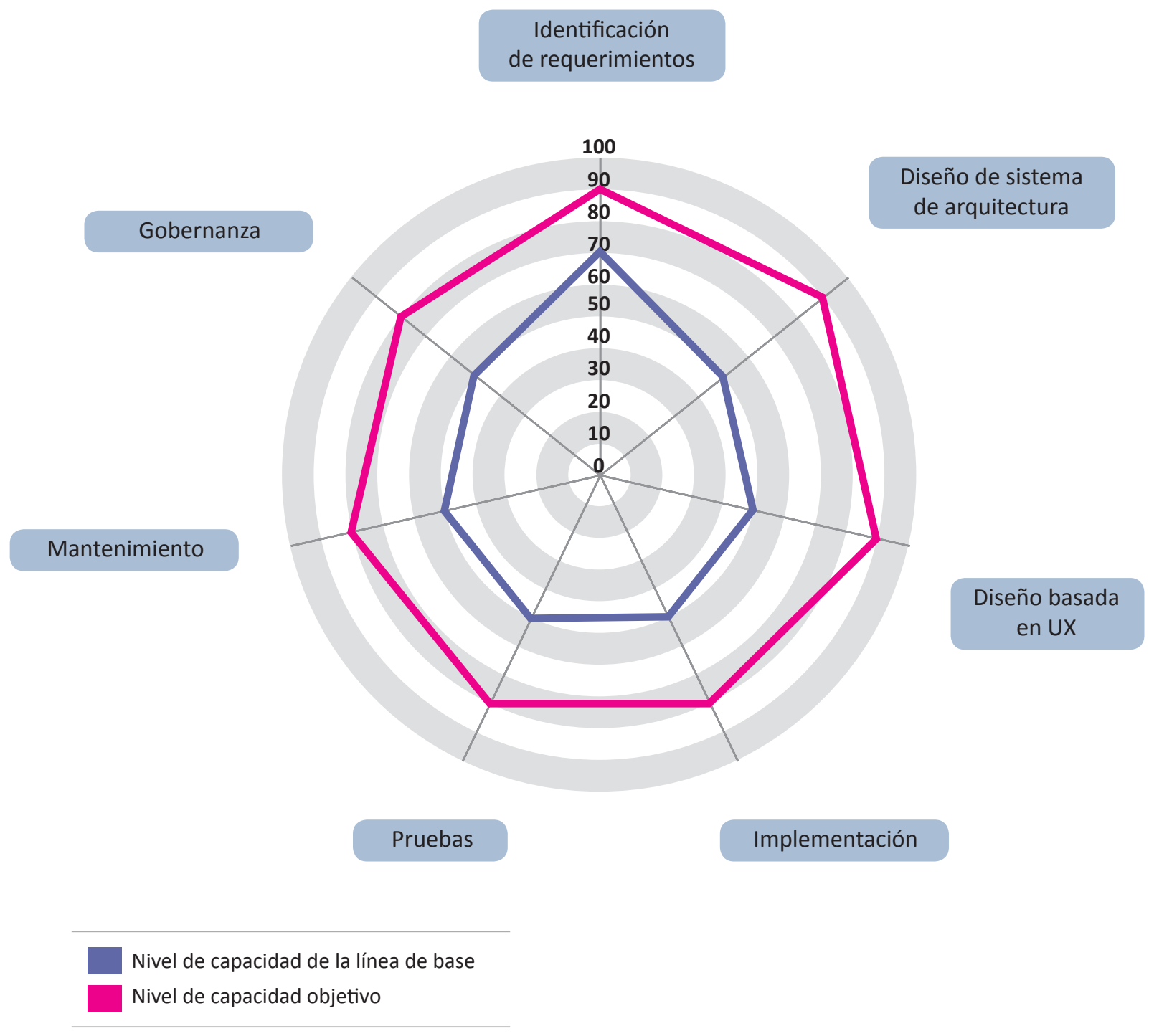

Fig. 5. Capacidades de TI para apoyar el desarrollo arquitectura. Fuente: Fuente propia.

Con relación a estas capacidades se encontraron las siguientes brechas: 20 puntos en identificación de requerimientos; 40 puntos en diseño de sistema de arquitectura; 40 puntos en diseño basado en la experiencia de usuario (UX); 30 puntos en implementación; 30 puntos en pruebas; 31 puntos en mantenimiento; 30 puntos en gobernanza. 


\section{C.Fase visión de arquitectura de negocio}

"Describe la estrategia de productos y servicios de la empresa, así como los aspectos organizacionales, funcionales, de procesos y de información" [9].
Para iniciar la visión de la arquitectura fue importante establecer los requisitos de dominio del negocio que se presentan en la tabla 2.
Requisito

Se requiere garantizar la calidad en los procedimientos de gestión curricular de la Escuela ECBTI, con la optimización del uso de recursos, tiempos y seguridad de la información presentada.

Capacidad de gestión de información digital.

Capacidad de asegurar la información.

Niveles de acceso a la información.

\section{Descripción}

Optimizar los recursos técnicos y humanos que permitan el control del ciclo de vida de los procedimientos de gestión curricular.

Se debe contar con recursos, talento humano y componentes que permitan gestionar de manera oportuna y apropiada la información.

Debe garantizar la seguridad, confiabilidad, disponibilidad y salvaguarda de los datos gestionados.

Se deben implementar políticas de acceso a la información, acompañado de registros de auditoria.

Tabla 2. Requisitos de dominio del negocio

Fuente: elaboración propia.

También se enumeran y describen los riesgos arquitectónicos, que son los temas o problemas que pueden surgir relacionados con el desarrollo de la arquitectura.

Los riesgos que se han producido o se han identificado se enumeran a continuación:

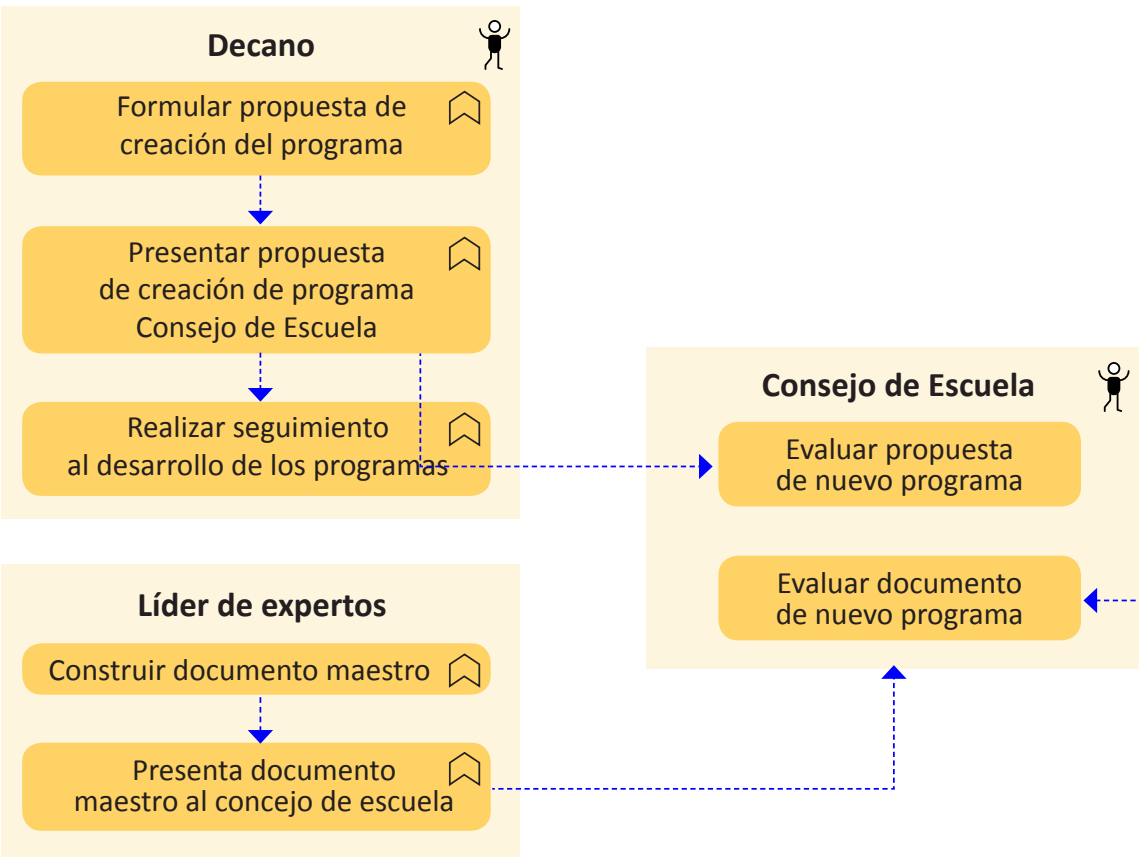

- Cambio repentino en normatividad.

- Rotación de personal calificado.

- No contar con recursos técnicos necesarios y suficientes.

- Cambios directrices de ECBTI.

En la figura 6 se identifica el nivel de función empresarial a partir del funcionamiento actual de la ECBTI.

Fig. 6. Nivel de función empresarial.

Fuente: Fuente propia. 
En la figura 7 se representan las arquitecturas de referencia y destino (Análisis GAP). En este diagrama se visualizan la adición, remoción y reordenación de componentes arquitectónicos, y en tabla 3 se hace el resumen de las diferencias en detalle.

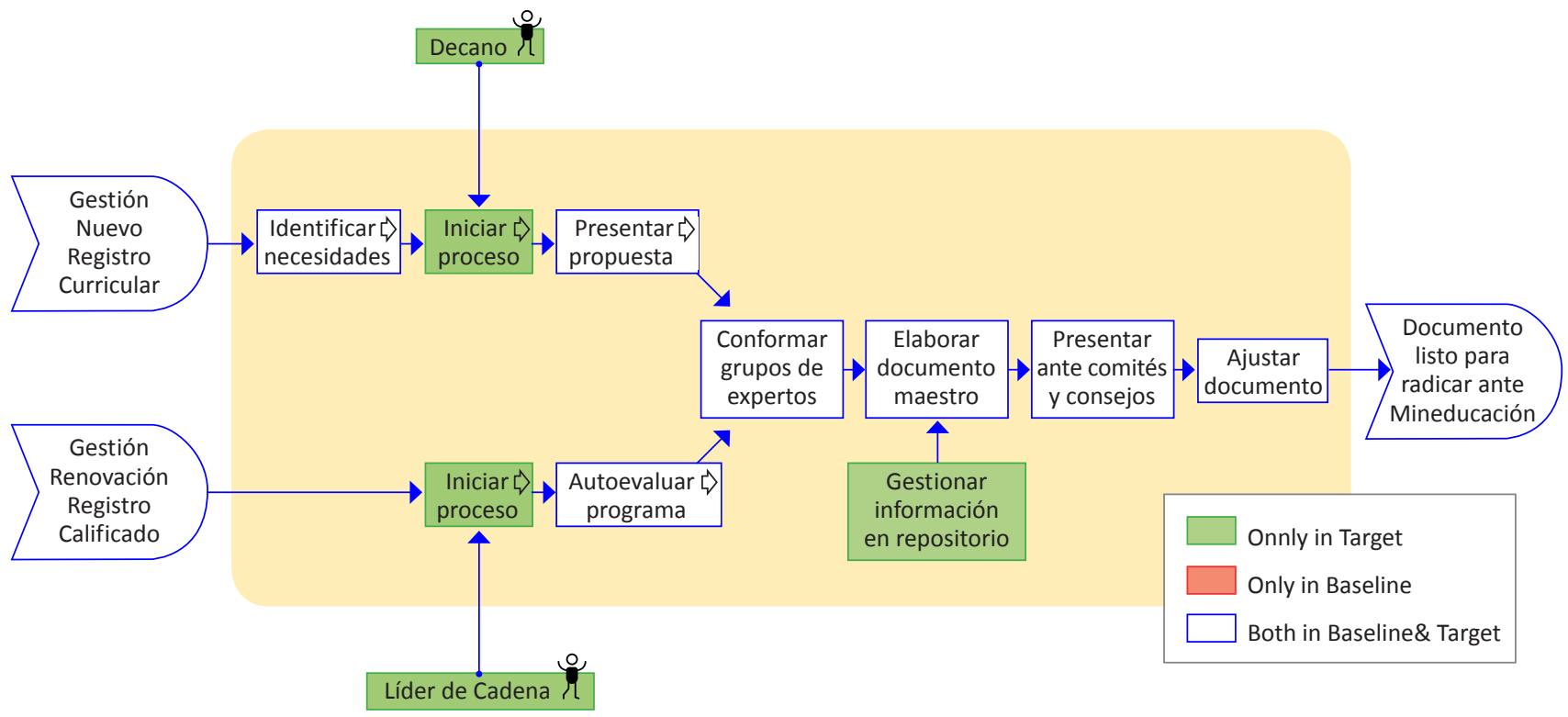

Figura 7. Análisis GAP.

Fuente: Fuente propia.

\begin{tabular}{|l|l|}
\hline Elementos de descripción & \multicolumn{1}{|c}{\begin{tabular}{c} 
Descripción de elementos para el proceso \\
\hline Gente
\end{tabular}} \\
\begin{tabular}{|l|l|} 
Los actores que intervienen en los procedimientos de acreditación \\
contarán con una herramienta de gestión curricular donde se registrará \\
la información de los programas en gestión o renovación, lo que permite \\
mantener una información centralizada y unificada para las personas que \\
intervienen, además de contar con un repositorio de documentos soporte.
\end{tabular} \\
\hline Proceso & $\begin{array}{l}\text { Los cronogramas de trabajo serán controlados de acuerdo con la } \\
\text { programación establecida y la información verificada. }\end{array}$ \\
\hline Herramientas & Integración de herramientas de colaboración y comunicación. \\
\hline Información & Información centralizada, segura, disponible y verificada. \\
\hline
\end{tabular}

Tabla 3. Descripción GAP.

Fuente: elaboración propia.

El análisis de impacto permite a la Escuela determinar el impacto del cambio requerido para la transición de la línea de base a la arquitectura de destino como son: impactos en arquitecturas preexistentes, cambios recientes que causan un impacto, oportunidades para aprovechar el trabajo, impactos, en otros proyectos.
A partir de esto y con la arquitectura de datos línea base, se logró diseñar la arquitectura de datos de destino (ver en la figura 8).

En la figura 8 se visualiza un modelo gráfico que representa la información arquitectónica del objetivo de los datos de arquitectura. 


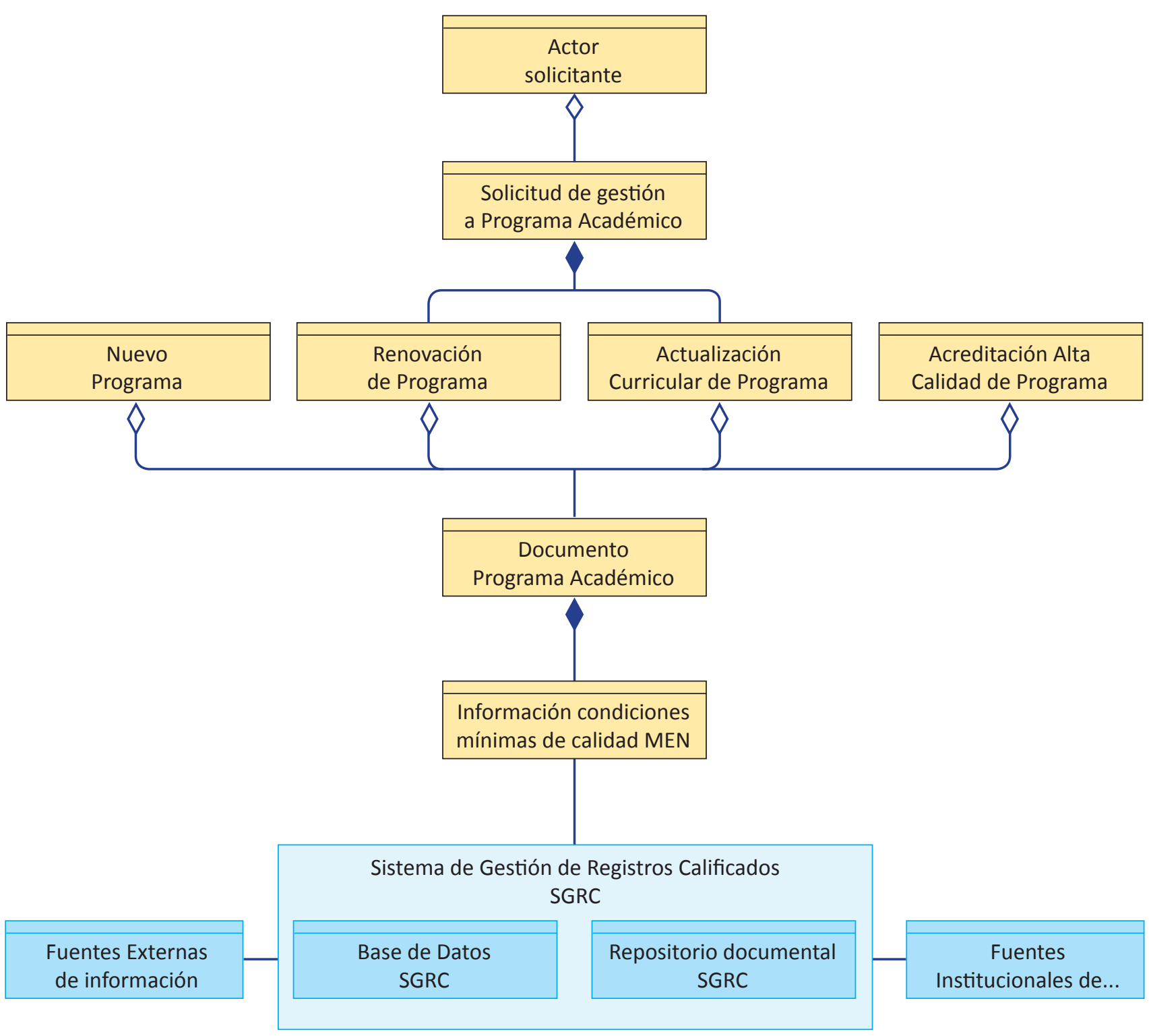

Fig. 8. Arquitectura de datos destino. Fuente: elaboración propia. 
En la figura 9 se representa la arquitectura de aplicaciones base con las que cuenta y funciona la ECBTI en específico para los procedimientos objeto de estudio.
A partir de esta estructura base se presenta lo que puede ser la arquitectura destino, como se puede observar en la figura 10.

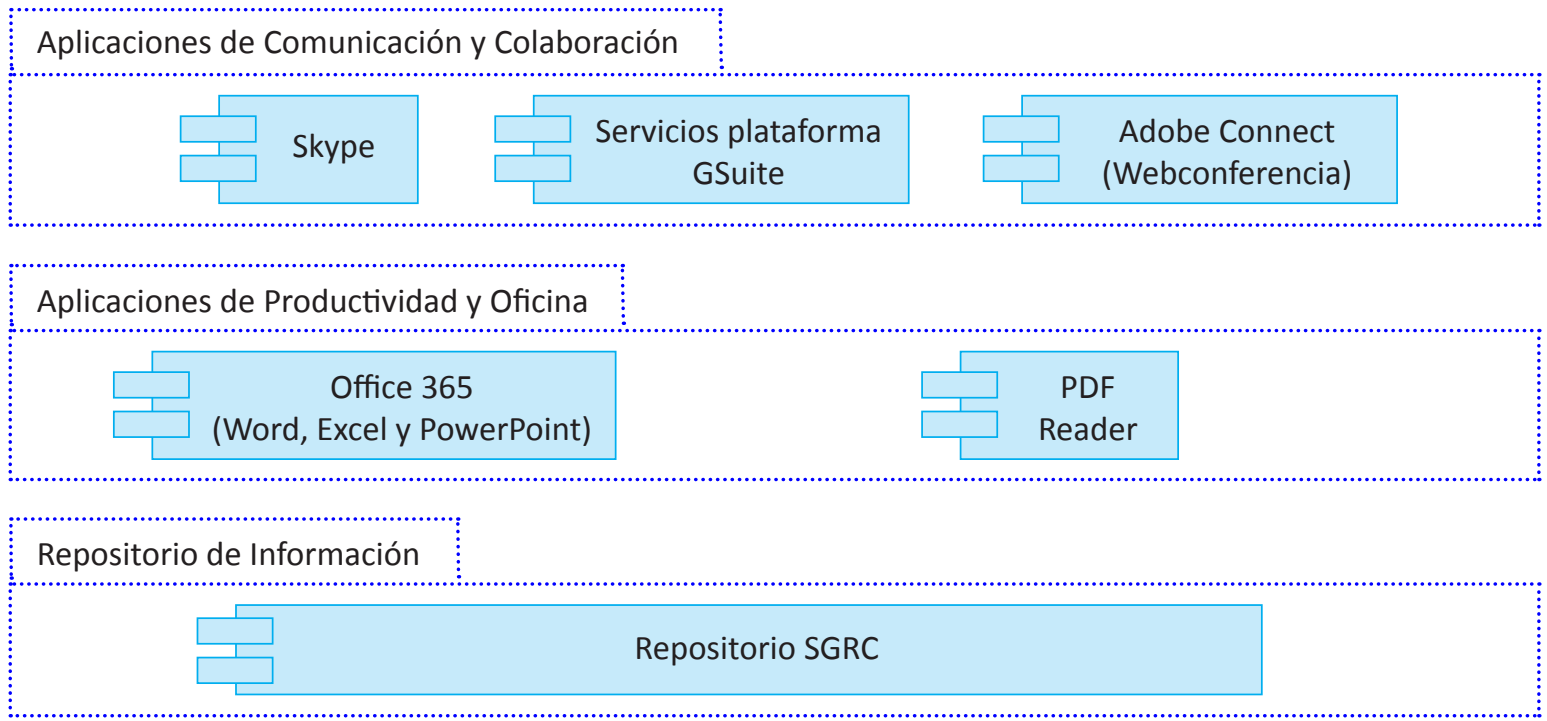

Fig. 9. Arquitectura de aplicación base.

Fuente: elaboración propia.

\section{Aplicaciones de Consulta Pública}

Portal de consulta

\section{Aplicaciones de Gestión Interna}

Aplicación SGRC
(Sistema de Gestión de Registros Curriculares)

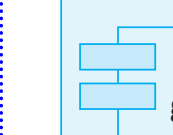

Componente de gestión de programas

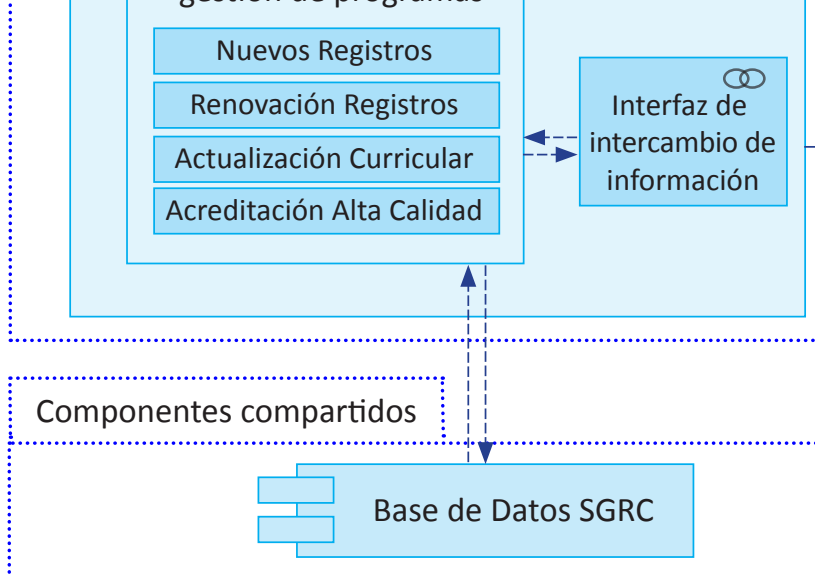

Fig. 10. Arquitectura de aplicación destino. Fuente: elaboración propia. 
Análisis de brechas entre la arquitectura de referencia y la de destino representadas en la figura 11 y 12. Contiene diagrama que visualiza la adición, remoción y reordenación de componentes arquitectónicos.

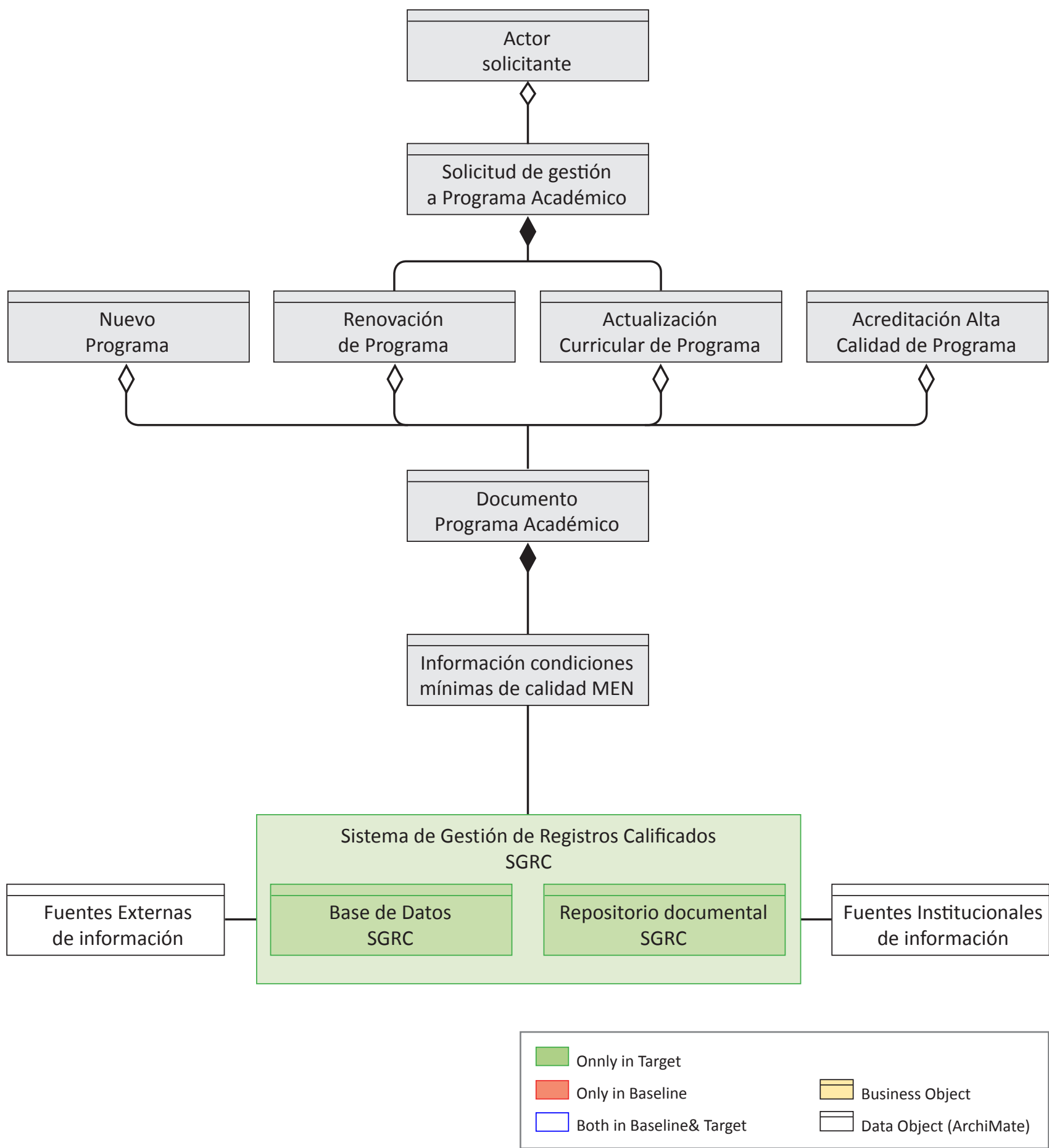

Fig. 11. Análisis GAP

Fuente: Fuente propia 


\section{D.Arquitectura de sistemas de información}

"La arquitectura de sistemas de información pretende dar a conocer cómo y dónde se almacena la información, cómo está relacionada, así como la seguridad que maneja” [10], por tanto, se indicará la línea base y la propuesta que se tiene para la información que almacena la ECBTI.

En la tabla 4 se presentan los requisitos de dominio de datos.

A partir de lo encontrado se propone una estructura para la información arquitectónica del objetivo datos arquitectura, como se pudo observar en la figura 8 .
Con lo anterior y sumando la arquitectura de aplicación base y la aplicación destino se realizó el análisis de brechas, que se pudo observar en la figura 12.

\section{\begin{tabular}{ll} 
Requisito $\quad$ Descripción \\
\hline
\end{tabular}}

Se requiere diseñar una estructura Concebir basada en base de datos que permita un diseño optimizar el rendimiento y reducir de base de la redundancia de datos, así mismo datos y de se requiere una organización de repositorio. la información administrada en el repositorio de archivos.

Tabla 4. Requisitos de dominio de datos

Fuente: elaboración propia.

\section{Aplicaciones de Consulta Pública}

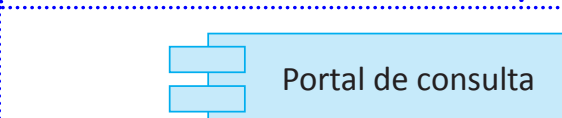

Aplicaciones de Gestión Interna

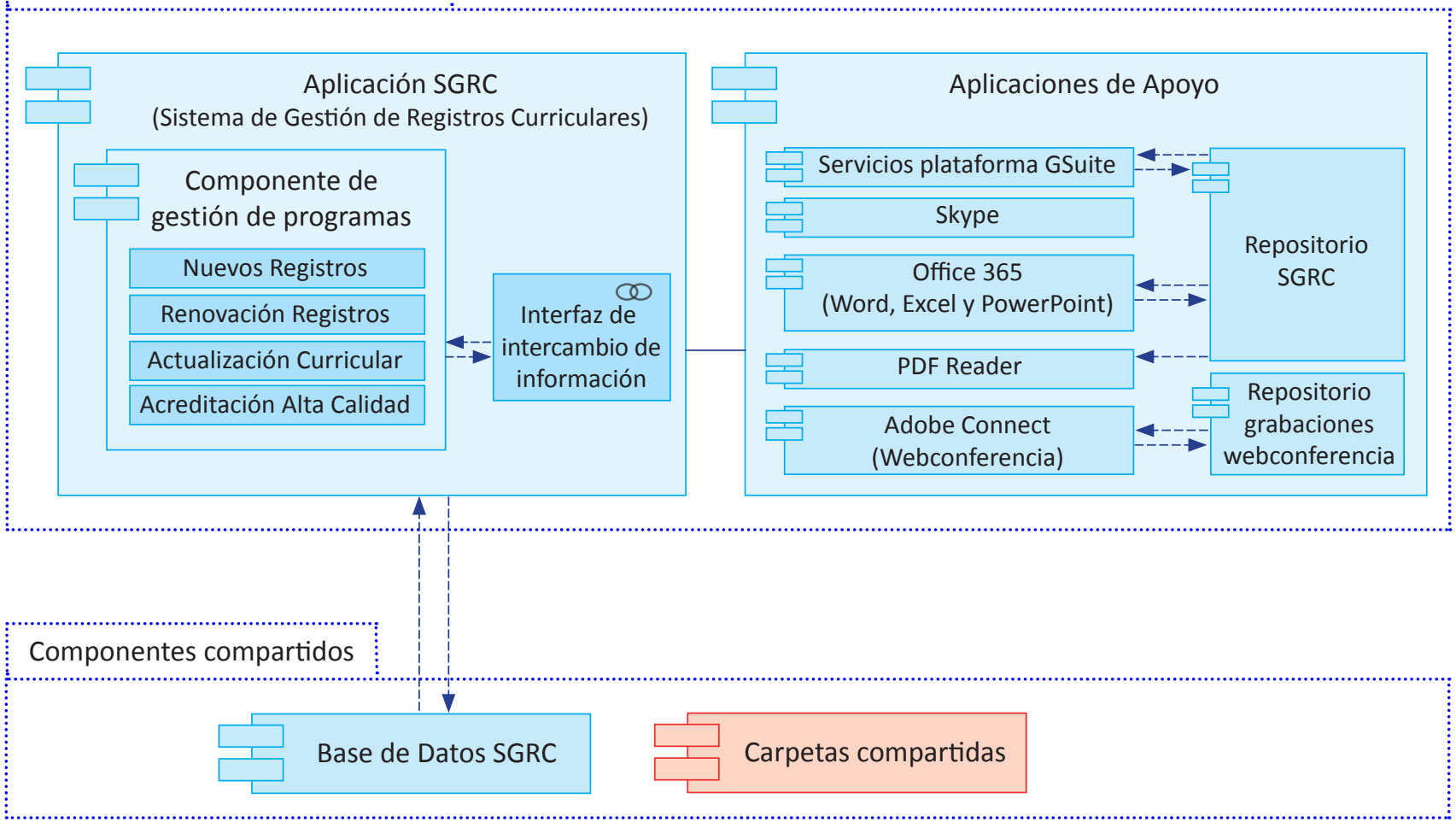

Fig. 12. Brecha dominio de aplicación.

Fuente: elaboración propia. 


\section{E. Arquitectura tecnológica}

"Define las capacidades de software y hardware que son requeridas para soportar los servicios y los procesos misionales de la empresa, incluye entre otros: infraestructura de $\mathrm{TI}$, redes, comunicaciones y estándares" [11].

En la tabla 5 se presenta la relación de los requerimientos de dominio a nivel tecnológico.

A partir de la arquitectura existente y como se representa en la figura 13 se hace la representación de la arquitectura que se propone como destino, con la cual se podrá realizar seguimiento adecuado a la gestión de la oferta educativa en sus procedimientos: nuevo registro calificado y renovación de registro ( $\mathrm{p}-8-1$ y $\mathrm{p}-8-3)$

\begin{tabular}{|l|l|}
\hline \multicolumn{1}{|c|}{ Requisito } & \multicolumn{1}{c|}{ Descripción } \\
\hline $\begin{array}{l}\text { Infraestructura de red } \\
\text { basada en PaaS. }\end{array}$ & $\begin{array}{l}\text { Gestionar la adquisición de la } \\
\text { infraestructura de red basado en } \\
\text { Plataforma como servicio PaaS, } \\
\text { para alojar las aplicaciones y } \\
\text { bases de datos de la ECBTI. }\end{array}$ \\
\hline $\begin{array}{l}\text { Garantizar el } \\
\text { sostenimiento de los } \\
\text { servicios en línea. }\end{array}$ & $\begin{array}{l}\text { Garantizar los recursos para } \\
\text { los servicios en la nube de } \\
\text { comunicación y colaboración. }\end{array}$ \\
\hline $\begin{array}{l}\text { Personal calificado para } \\
\text { soporte. }\end{array}$ & $\begin{array}{l}\text { Garantizar el personal soporte } \\
\text { para el apoyo a los usuarios } \\
\text { finales en la aplicación SGRC. }\end{array}$ \\
\hline
\end{tabular}

Tabla 5. Requisitos de dominio tecnológico

Fuente: elaboración propia.

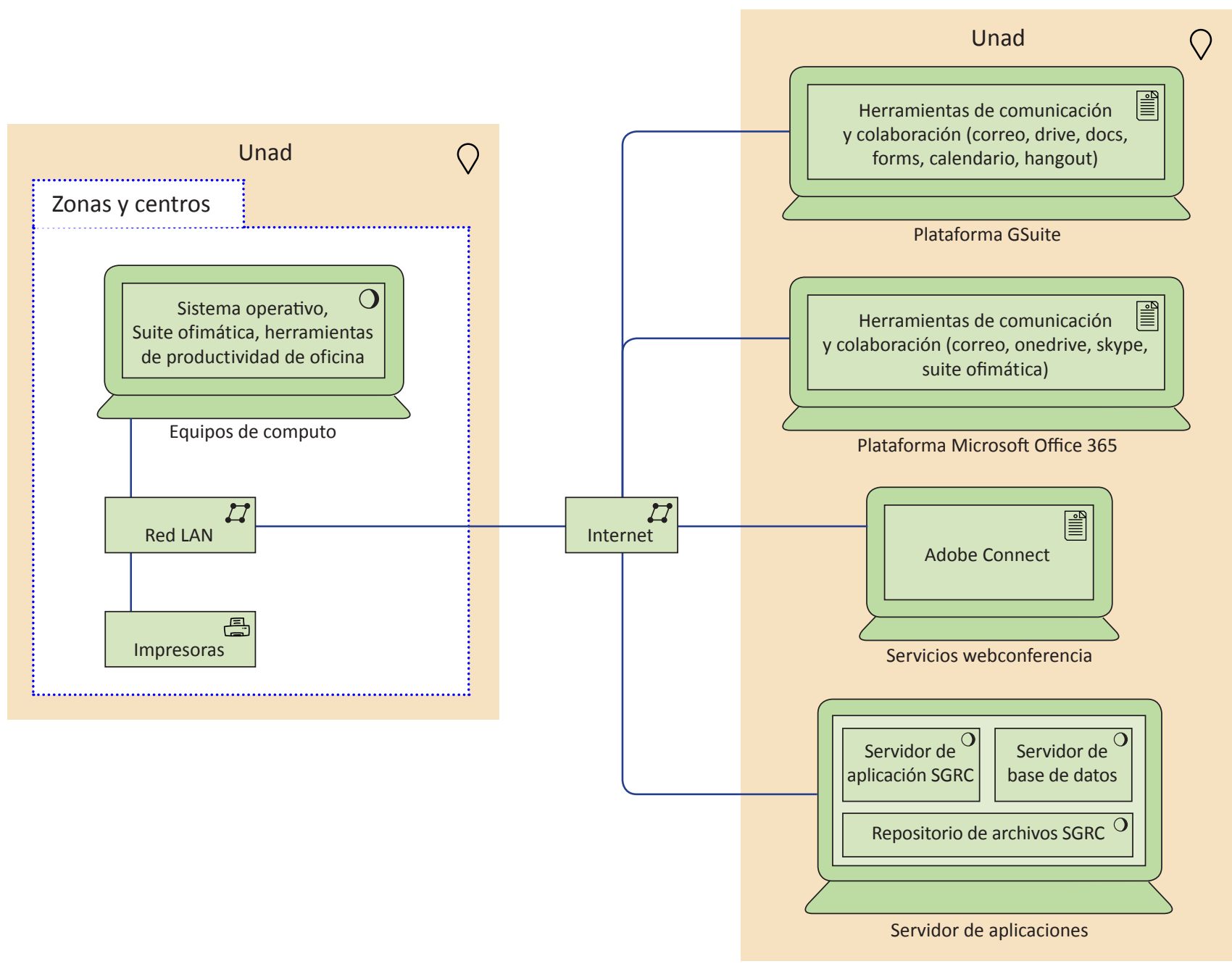

Fig. 13. Arquitectura destino.

Fuente: elaboración propia. 


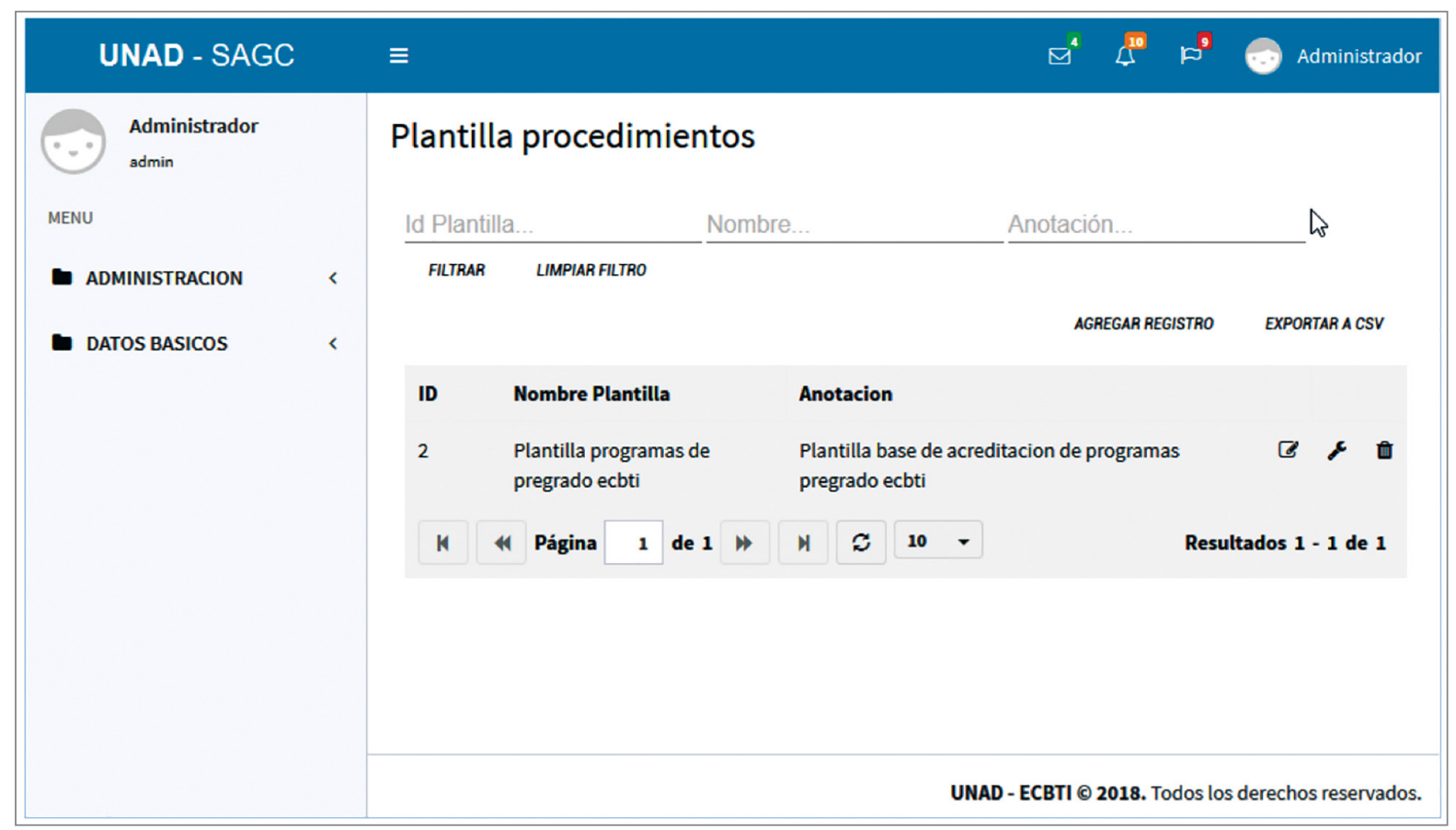

Fig. 14. Vista de la aplicación.

Fuente: Elaboración propia.

Para el momento de la escritura del presente artículo ya se ha avanzado en la construcción de una aplicación que permita apoyar la gestión de la oferta educativa, una vista de esta se pude consultar en la figura 14.

Esta aplicación permitirá continuar con las fases siguientes de ADM [12] y poder validar las propuestas presentadas.

\section{IV.CONCLUSIONES}

La arquitectura empresarial es una estrategia como solución a la integración de la arquitectura tecnológica, sistemas de información y procesos de negocios. Implementar la arquitectura empresarial no se trata de establecer un conjunto de aplicaciones de servicios informáticos, ni tampoco se trata de integrar aplicaciones del negocio, sino que va más allá, debe integrar los sistemas a los procesos del negocio de forma que genere una ventaja competitiva y se convierta en una estrategia de la ECBTI.

Una revisión de las capacidades de la ECBTI basada en los procesos de negocio para la gestión de la oferta educativa permitió un análisis desde una vista de bajo nivel de cómo funciona la Escuela, mediante la identificación de procesos
(Procesos AS-IS), lo que permite proponer mejoras y optimizar los procesos bajo un análisis no manual sino con una visión automatizada (Procesos TO BE), que posibilitarán una implementar una arquitectura global.

La arquitectura empresarial propuesta permitió identificar la relación entre los objetivos y las estrategias de la Escuela, y a partir de ello se encuentra que existe la tecnología necesaria, pero se requiere un trabajo fuerte en los aspectos organizacionales [13], [14].

\section{REFERENCIAS}

[1] Togaf. (2013). “http://pubs.opengroup.org,". [En línea]. Disponible en: http://pubs.opengroup.org/architecture/togaf 8-doc/arch/ chap30.html

[2] H. Cruz Bueno y W. Briceño Pineda. (2015). "Identificación de principios de arquitectura empresarial para la gestión de factores de impacto en entidades públicas colombianas utilizando Togaf",. Inventum. Vol. 10, $\mathrm{n}^{\circ} 18$, pp. 22-28. [En línea]. Disponible en: https:// revistas.uniminuto.edu/index.php/Inventum/ article/view/1216. 
[3] Togaf 9.2. (2016). "The Open Group" 2016. [En línea]. Disponible en: http://pubs.opengroup. org/architecture/togaf9-doc/arch/.

[4] A. Josey. (2013). "The open group," 2013. [En línea]. Disponible en: https://bit.ly/2ZpMwlR

[5] R. Canabal y C. Amaury. (2017). “Aplicación de un Esquema de Arquitectura Empresarial (Togaf) para una Pequeña Empresa (PYME) utilizando Aplicaciones Colaborativas de Google" Información Tecnológica. Vol. 28, $\mathrm{n}^{\circ} 4$, pp. 8592. [En línea]. Disponible en: https://scielo. conicyt.cl/pdf/infotec/v28n4/art11.pdf.

[6] D. F. Ruiz, Diseño de arquitectura empresarial en el sector educativo colombiano: caso colegio privado en Bogotá, Trabajo de grado, Programa de Ingeniería de Sistemas, Universidad Católica de Colombia, Bogotá, 2014.

[7] N. Saboya Rios y L. Jara. (2018). "Biblioteca Unad”. [En línea]. Disponible en: https: / /www. redalyc.org/jatsRepo/4676/467655911004/ html/index.html

[8] SIG, 2015. "Sistema integrado de gestión". [En línea]. Disponible en: https://sig.unad.edu. $\mathrm{co} /$.

[9] E. Arroyo. (2018). "Definición de principios de arquitectura para arquitectura empresarial de la organización" [En línea]. Disponible en: https: / / ecorfan.org/handbooks/Ciencias-TIT_I/IBERO-Handbook_MR_1-43-52.pdf
[10] E. Álvarez, Arquitectura Empresarial para la Dirección de Tecnología de Información y Comunicación de la Universidad de Cuenca, Trabajo de graduación previo a la obtención del grado de magister en gestión estratégica de tecnologías de la información. Facultad de Ingeniería, Cuenca, Universidad de Cuenca, Cuenca, 2017.

[11] A. Gualteros, Modelo de Arquitectura Empresarial para Empresas Innovadoras en el Sector de Telecomunicaciones (TELCO), Trabajo final de maestría presentado como requisito parcial para optar al título de: Magister en Ingeniería de Sistemas y Computación. Facultad de Ingeniería, Universidad Nacional de Colombia, Bogotá, 2017.

[12] Visual Parading. (2014, Mayo 4). "Togaf ADM Software:Act and GenerateADM Deliverables". [En línea]. Disponible en: https: / /www.visualparadigm.com/tutorials/togaf-adm-toolfor-enterprise-architecture.jsp.

[13] GeneXus. (2013, Octubre 12). "Mejorando la gestión de gerencia de TI: Togaf en la gestión de arquitecturas de TI de ANCAP". [En línea]. Disponible en: https://es.slideshare.net/ge nexus/127-mejorando-lagestiondelageren ciadeti.

[14] MinTic. (2014, Junio 17). "MinTIC da a conocer borrador de Arquitectura Empresarial (AE) para Gestión de TI en el Estado". [En línea]. Disponible en: https: / /www.mintic.gov. $\mathrm{co} /$ portal/604/w3-article-6313.html. 